\title{
Fractional Complex Transform and exp-Function Methods for Fractional Differential Equations
}

\author{
Ahmet Bekir, ${ }^{1}$ Özkan Güner, ${ }^{2}$ and Adem C. Cevikel ${ }^{3}$ \\ ${ }^{1}$ Eskisehir Osmangazi University, Art-Science Faculty, Department of Mathematics and Computer Science, 26480 Eskişehir, Turkey \\ ${ }^{2}$ Dumlupinar University, School of Applied Sciences, Department of Management Information Systems, 43100 Kütahya, Turkey \\ ${ }^{3}$ Yildiz Technical University, Education Faculty, Department of Mathematics Education, 34220 Istanbul, Turkey
}

Correspondence should be addressed to Adem C. Cevikel; acevikel@yildiz.edu.tr

Received 1 March 2013; Accepted 21 March 2013

Academic Editor: Mustafa Bayram

Copyright (C) 2013 Ahmet Bekir et al. This is an open access article distributed under the Creative Commons Attribution License, which permits unrestricted use, distribution, and reproduction in any medium, provided the original work is properly cited.

\begin{abstract}
The exp-function method is presented for finding the exact solutions of nonlinear fractional equations. New solutions are constructed in fractional complex transform to convert fractional differential equations into ordinary differential equations. The fractional derivatives are described in Jumarie's modified Riemann-Liouville sense. We apply the exp-function method to both the nonlinear time and space fractional differential equations. As a result, some new exact solutions for them are successfully established.
\end{abstract}

\section{Introduction}

Fractional differential equations (FDEs) are viewed as alternative models to nonlinear differential equations. Varieties of them play important roles and serve as tools not only in mathematics but also in physics, biology, fluid flow, signal processing, control theory, systems identification, and fractional dynamics to create the mathematical modeling of many nonlinear phenomena. Besides, they are employed in social sciences such as food supplement, climate, finance, and economics. Oldham and Spanier first considered the fractional differential equations arising in diffusion problems [1]. The fractional differential equations have been investigated by many authors [2-4].

In recent decades, some effective methods for fractional calculus appeared in open literature, such as the exp-function method [5], the fractional subequation method [6-8], the $\left(G^{\prime} / G\right)$-expansion method $[9,10]$, and the first integral method [11].

The fractional complex transform $[12,13]$ is the simplest approach; it is to convert the fractional differential equations into ordinary differential equations, making the solution procedure extremely simple. Recently, the fractional complex transform has been suggested to convert fractional-order differential equations with modified
Riemann-Liouville derivatives into integer order differential equations, and the reduced equations can be solved by symbolic computation. The exp-function method [14-20] can be used to construct the exact solutions for fractional differential equations. The present paper investigates the applicability and efficiency of the exp-function method on fractional nonlinear differential equations. The aim of this paper is to extend the application of the exp-function method to obtain exact solutions to some fractional differential equations in mathematical physics and biology.

This paper is organized as follows. In Section 2, some basic properties of Jumarie's modified Riemann-Liouville derivative are given. The main steps of the exp-function method are given in Section 3. In Sections 4-6, we construct the exact solutions of the fractional-order biological population model, fractional Burgers equation, and fractional Cahn-Hilliard equation via this method. Some conclusions are shown in Section 7.

\section{Modified Riemann-Liouville Derivative}

In the last few decades, in order to improve the local behavior of fractional types, a few local versions of fractional derivatives have been proposed, that is, Caputo's fractional derivative [21], Grünwald-Letnikov's fractional derivative [22], 
the Riemann-Liouville derivative [22], Jumarie's modified Riemann-Liouville derivative $[23,24]$. Jumarie's derivative is defined as

$$
\begin{aligned}
& D_{t}^{\alpha} f(t) \\
& \quad=\frac{1}{\Gamma(1-\alpha)} \frac{d}{d t} \int_{0}^{t}(t-\xi)^{-\alpha}[f(\xi)-f(0)] d \xi, \quad 0<\alpha<1,
\end{aligned}
$$

where $f: R \rightarrow R, t \rightarrow f(t)$ denotes a continuous (but not necessarily first-order-differentiable) function. We list some important properties for the modified Riemann-Liouville derivative as follows.

(1) Assume that $f(t)$ denotes a continuous $R \rightarrow R$ function. We use the following equality for the integral with respect to $(d t)^{\alpha}$ :

$$
\begin{aligned}
& I_{t}^{\alpha} f(t)=\frac{1}{\Gamma(\alpha)} \int_{0}^{t}(t-\xi)^{\alpha-1} f(\xi) d \xi, \\
& I_{t}^{\alpha} f(t)=\frac{1}{\Gamma(\alpha+1)} \int_{0}^{t} f(\xi)(d t)^{\alpha}, \quad 0<\alpha \leq 1 .
\end{aligned}
$$

(2) Some useful formulas include

$$
\begin{gathered}
f^{(\alpha)}[g(t)]=\frac{d f}{d t} g^{(\alpha)}(t), \\
D_{t}^{\alpha} t^{\gamma}=\frac{\Gamma(1+\gamma)}{\Gamma(1+\gamma-\alpha)} t^{\gamma-\alpha}, \\
\int(d t)^{\beta}=t^{\beta} .
\end{gathered}
$$

(3) Let $u(t)$ and $v(t)$ satisfy the definition of the modified Riemann-Liouville derivative, and let $f(t)$ be an $\alpha$ order-differentiable function:

$$
\begin{aligned}
D_{t}^{\alpha} & (u(t) v(t)) \\
\quad & =v(t) D_{t}^{\alpha} u(t)+u(t) D_{t}^{\alpha} v(t), \\
D_{t}^{\alpha} & f[u(t)] \\
\quad & f_{u}^{\prime}[u(t)] D_{t}^{\alpha} u(t)=D_{u}^{\alpha} f[u(t)]\left(u^{\prime}(t)\right)^{\alpha} .
\end{aligned}
$$

Function $f(t)$ should be differentiable with respect to $g(t)$, and $g(t)$ is fractional differentiable in (3). The previous results are employed in the following sections.

\section{Fractional Complex Transform and exp- Function Method}

We consider the following nonlinear FDE of the type

$$
\begin{array}{r}
F\left(u, D_{t}^{\alpha} u, D_{x}^{\beta} u, D_{t}^{\alpha} D_{t}^{\alpha} u, D_{t}^{\alpha} D_{x}^{\beta} u, D_{x}^{\beta} D_{x}^{\beta} u, \ldots\right)=0, \\
0<\alpha, \beta<1,
\end{array}
$$

where $u$ is an unknown function and $F$ is a polynomial of $u$ and its partial fractional derivatives, in which the highest order derivatives and the nonlinear terms are involved. In the following, we give the main steps of the exp-function method.

Step 1. Li and He $[25,26]$ suggested a fractional complex transform to convert fractional differential equations into ordinary differential equations, so all analytical methods devoted to the advanced calculus can be easily applied to the fractional calculus. The complex wave variable was as follows:

$$
\begin{gathered}
u(x, t)=U(\xi), \\
\xi=\frac{\tau x^{\beta}}{\Gamma(1+\beta)}+\frac{\lambda t^{\alpha}}{\Gamma(1+\alpha)},
\end{gathered}
$$

where $\tau$ and $\lambda$ are nonzero arbitrary constants; we can rewrite (7) in the following nonlinear ordinary differential equation:

$$
Q\left(U, U^{\prime}, U^{\prime \prime}, U^{\prime \prime \prime}, \ldots\right)=0,
$$

where the prime denotes the derivation with respect to $\xi$. If possible, we should integrate (9) term by term one or more times.

Step 2. According to exp-function method, which was developed by $\mathrm{He}$ and $\mathrm{Wu}[14]$, we assume that the wave solution can be expressed in the following form:

$$
U(\xi)=\frac{\sum_{n=-c}^{d} a_{n} \exp [n \xi]}{\sum_{m=-p}^{q} b_{m} \exp [m \xi]},
$$

where $p, q, c$, and $d$ are positive integers which are known to be further determined and $a_{n}$ and $b_{m}$ are unknown constants. We can rewrite (10) in the following equivalent form:

$$
U(\xi)=\frac{a_{-c} \exp [-c \xi]+\cdots+a_{d} \exp [d \xi]}{b_{-p} \exp [-p \xi]+\cdots+b_{q} \exp [q \xi]} .
$$

Step 3. This equivalent formulation plays a significant and fundamental part for finding the exact solution of mathematical problems. To determine the values of $c$ and $p$, we balance the linear term of highest order of (9) with the highest order nonlinear term. Similarly, to determine the value of $d$ and $q$, we balance the linear term of lowest order of (9) with lowest order nonlinear term [27-29].

In the following sections, we present three examples to illustrate the applicability of the exp-function method and fractional complex transform to solve nonlinear fractional differential equations.

\section{Fractional-Order Biological Population Model}

We consider a time fractional biological population model of the form $[30,31]$

$$
\begin{array}{r}
\frac{\partial^{\alpha} u}{\partial t^{\alpha}}=\frac{\partial^{2}}{\partial x^{2}}\left(u^{2}\right)+\frac{\partial^{2}}{\partial y^{2}}\left(u^{2}\right)+h\left(u^{2}-r\right), \quad t>0 \\
0<\alpha \leq 1, x, y \in R
\end{array}
$$


where $u$ denotes the population density $h\left(u^{2}-r\right)$ represents the population supply due to births and deaths, and $h, r$ are constants.

For our goal, we present the following transformation:

$$
u(x, y, t)=U(\xi), \quad \xi=v x+i v y-\frac{c t^{\alpha}}{\Gamma(1+\alpha)},
$$

where $c$ and $v$ are constants and $i^{2}=-1$.

Then by the use of (13), (12) can be turned into an ODE:

$$
c U^{\prime}+h U^{2}-h r=0,
$$

where " $U^{\prime \prime}=d U / d \xi$.

Balancing the order of $U^{\prime}$ and $U^{2}$ in (14), we get

$$
\begin{gathered}
U^{\prime}=\frac{c_{1} \exp [-(c+p) \xi]+\cdots}{c_{2} \exp [-2 p \xi]+\cdots}, \\
U^{2}=\frac{c_{3} \exp [-2 c \xi]+\cdots}{c_{4} \exp [-2 p \xi]+\cdots},
\end{gathered}
$$

where $c_{i}$ are determined coefficients only for simplicity. Balancing highest order of exp-function in (15), we obtain

$$
-(p+c)=-2 c,
$$

which leads to the result that

$$
p=c .
$$

In the same way to determine the values of $d$ and $q$, we balance the linear term of the lowest order in (14):

$$
\begin{gathered}
U^{\prime}=\frac{\cdots+d_{1} \exp [(q+d) \xi]}{\cdots+d_{2} \exp [2 q \xi]}, \\
U^{2}=\frac{\cdots+d_{3} \exp [2 d \xi]}{\cdots+d_{4} \exp [2 q \xi]},
\end{gathered}
$$

where $d_{i}$ are determined coefficients only for simplicity. From (18), we have

$$
q+d=2 d,
$$

and this gives

$$
q=d
$$

For simplicity, we set $p=c=1$ and $q=d=1$, so (11) reduces to

$$
U(\xi)=\frac{a_{1} \exp (\xi)+a_{0}+a_{-1} \exp (-\xi)}{b_{1} \exp (\xi)+b_{0}+b_{-1} \exp (-\xi)} .
$$

Substituting (21) into (14) and by the help of symbolic computation, we have

$$
\begin{gathered}
\frac{1}{A}\left[R_{2} \exp (2 \xi)+R_{1} \exp (\xi)+R_{0}+R_{-1} \exp (-\xi)\right. \\
\left.+R_{-2} \exp (-2 \xi)\right]=0,
\end{gathered}
$$

where

$$
\begin{aligned}
A= & \left(b_{-1} \exp (-\xi)+b_{0}+b_{1} \exp (\xi)\right)^{2}, \\
R_{2}= & h a_{1}^{2}-h r b_{1}^{2}, \\
R_{1}= & c a_{1} b_{0}-c a_{0} b_{1}+2 h a_{1} a_{0}-2 h r b_{1} b_{0}, \\
R_{0}= & -2 c a_{-1} b_{1}+2 c a_{1} b_{-1}-2 h r b_{1} b_{-1}-h r b_{0}^{2} \\
& +2 h a_{1} a_{-1}+h a_{0}^{2}, \\
R_{-1}= & -c a_{-1} b_{0}+c a_{0} b_{-1}+2 h a_{0} a_{-1}-2 h r b_{0} b_{-1}, \\
R_{-2}= & h a_{-1}^{2}-h r b_{-1}^{2} .
\end{aligned}
$$

Solving this system of algebraic equations by using symbolic computation, we get the following results.

Case 1. Consider

$$
\begin{array}{ll}
a_{1}=\frac{r b_{0}^{2}-a_{0}^{2}}{4 \sqrt{r} b_{-1}}, & a_{0}=a_{0}, \\
b_{1}=\frac{r b_{0}^{2}-a_{0}^{2}}{4 r b_{-1}}, & a_{-1}=-\sqrt{r} b_{-1}, \\
c=2 h \sqrt{r}, &
\end{array}
$$

where $a_{0}, b_{0}$, and $b_{-1}$ are free parameters which exist provided that $b_{-1} \neq 0$. Substituting these results into (21), we get the following exact solution:

$$
\begin{aligned}
& u(x, y, t) \\
& =\frac{r b_{0}^{2}-a_{0}^{2}}{4 \sqrt{r} b_{-1}} \exp \left(v x+i v y-\frac{2 h \sqrt{r} t^{\alpha}}{\Gamma(1+\alpha)}\right) \\
& +a_{0}-\sqrt{r} b_{-1} \exp \left(-\left(v x+i v y-\frac{2 h \sqrt{r} t^{\alpha}}{\Gamma(1+\alpha)}\right)\right) \\
& \quad \times\left(\frac{r b_{0}^{2}-a_{0}^{2}}{4 r b_{-1}} \exp \left(v x+i v y-\frac{2 h \sqrt{r} t^{\alpha}}{\Gamma(1+\alpha)}\right)\right. \\
& \left.\quad+b_{0}+b_{-1} \exp \left(-\left(v x+i v y-\frac{2 h \sqrt{r} t^{\alpha}}{\Gamma(1+\alpha)}\right)\right)\right)^{-1} .
\end{aligned}
$$

Case 2. Consider

$$
\begin{aligned}
& a_{1}=\frac{a_{0}^{2}}{4 \sqrt{r} b_{-1}}, \quad a_{0}=a_{0}, \quad a_{-1}=-\sqrt{r} b_{-1}, \\
& b_{1}=-\frac{a_{0}^{2}}{4 r b_{-1}}, \quad b_{0}=0, \quad b_{-1}=b_{-1} \text {, } \\
& c=2 h \sqrt{r},
\end{aligned}
$$


where $a_{0}$ and $b_{-1}$ are free parameters, which exist provided that $b_{-1} \neq 0$. Substituting these results into (21), we obtain the following exact solution:

$$
\begin{aligned}
& u(x, y, t) \\
& =\frac{a_{0}^{2}}{4 \sqrt{r} b_{-1}} \exp \left(v x+i v y-\frac{2 h \sqrt{r} t^{\alpha}}{\Gamma(1+\alpha)}\right)+a_{0} \\
& \quad-\sqrt{r} b_{-1} \exp \left(-\left(\nu x+i v y-\frac{2 h \sqrt{r} t^{\alpha}}{\Gamma(1+\alpha)}\right)\right) \\
& \quad \times\left(-\frac{a_{0}^{2}}{4 r b_{-1}} \exp \left(v x+i v y-\frac{2 h \sqrt{r} t^{\alpha}}{\Gamma(1+\alpha)}\right)\right. \\
& \left.\quad+b_{-1} \exp \left(-\left(\nu x+i v y-\frac{2 h \sqrt{r} t^{\alpha}}{\Gamma(1+\alpha)}\right)\right)\right)^{-1} .
\end{aligned}
$$

\section{Time Fractional Burgers Equation}

We consider the one-dimensional time fractional Burgers equation with the value problem [32]

$$
\begin{gathered}
\frac{\partial^{\alpha} u}{\partial t^{\alpha}}+\varepsilon u \frac{\partial u}{\partial x}-v \frac{\partial^{2} u}{\partial x^{2}}=0, \quad t>0,0<\alpha \leq 1, \\
u(x, 0)=g(x)
\end{gathered}
$$

where $\alpha$ is a parameter describing the order of the fractional time derivative. The function $u(x, t)$ is assumed to be a causal function of time.

For our purpose, we introduce the following transformations:

$$
u(x, t)=U(\xi), \quad \xi=\lambda x-\frac{c t^{\alpha}}{\Gamma(1+\alpha)}
$$

where $\lambda$ and $c$ are nonzero constants.

Substituting (30) into (28), we can show that (28) reduced into the following ODE:

$$
-c U^{\prime}+\lambda \varepsilon U U^{\prime}-\lambda^{2} v U^{\prime \prime}=0
$$

where " $U^{\prime \prime}=d U / d \xi$.

Integrating (31) with respect to $\xi$ yields

$$
-c U+\frac{\lambda \varepsilon}{2} U^{2}-\lambda^{2} \nu U^{\prime}+\xi_{0}=0,
$$

where $\xi_{0}$ is a constant of integration.

By the same procedure as illustrated in Section 3, we can determine values of $c$ and $p$ by balancing terms $U^{2}$ and $U^{\prime}$ in (32). By symbolic computation, we have

$$
\begin{gathered}
U^{\prime}=\frac{c_{1} \exp [-(c+p) \xi]+\cdots}{c_{2} \exp [-2 p \xi]+\cdots}, \\
U^{2}=\frac{c_{3} \exp [-2 c \xi]+\cdots}{c_{4} \exp [-2 p \xi]+\cdots},
\end{gathered}
$$

where $c_{i}$ are determined coefficients only for simplicity. Balancing the highest order of exp-function in (33), we have

$$
-(p+c)=-2 c
$$

which leads to the result that

$$
p=c \text {. }
$$

Similarly, to determine the values of $d$ and $q$, we balance the linear term of the lowest order in (32):

$$
\begin{gathered}
U^{\prime}=\frac{\cdots+d_{1} \exp [(q+d) \xi]}{\cdots+d_{2} \exp [2 q \xi]}, \\
U^{2}=\frac{\cdots+d_{3} \exp [2 d \xi]}{\cdots+d_{4} \exp [2 q \xi]},
\end{gathered}
$$

where $d_{i}$ are determined coefficients only for simplicity. From (36), we obtain

$$
q+d=2 d
$$

and this gives

$$
q=d
$$

For simplicity, we set $p=c=1$ and $q=d=1$, so (11) reduces to

$$
U(\xi)=\frac{a_{1} \exp (\xi)+a_{0}+a_{-1} \exp (-\xi)}{b_{1} \exp (\xi)+b_{0}+b_{-1} \exp (-\xi)} .
$$

Substituting (39) into (32) and by the help of symbolic computation, we have

$$
\begin{aligned}
& \frac{1}{A}\left[R_{2} \exp (2 \xi)+R_{1} \exp (\xi)+R_{0}\right. \\
& \left.\quad+R_{-1} \exp (-\xi)+R_{-2} \exp (-2 \xi)\right]=0
\end{aligned}
$$

where

$$
\begin{aligned}
A= & \left(b_{-1} \exp (-\xi)+b_{0}+b_{1} \exp (\xi)\right)^{2}, \\
R_{2}= & \xi_{0} b_{1}^{2}-c a_{1} b_{1}+\frac{1}{2} \lambda \varepsilon a_{1}^{2}, \\
R_{1}= & -\lambda^{2} \nu a_{1} b_{0}+\lambda \varepsilon a_{1} a_{0}+\lambda^{2} v a_{0} b_{1}-c a_{0} b_{1}-c a_{1} b_{0} \\
& +2 \xi_{0} b_{1} b_{0}, \\
R_{0}= & -2 \lambda^{2} v a_{1} b_{-1}+2 \lambda^{2} v a_{-1} b_{1}-c a_{0} b_{0}+\lambda \varepsilon a_{1} a_{-1} \\
& +\xi_{0} b_{0}^{2}-c a_{1} b_{-1}-c a_{-1} b_{1}+\frac{1}{2} \lambda \varepsilon a_{0}^{2}+2 \xi_{0} b_{1} b_{-1}, \\
R_{-1}= & \lambda \varepsilon a_{0} a_{-1}+\lambda^{2} \nu a_{-1} b_{0}-\lambda^{2} v a_{0} b_{-1}+2 \xi_{0} b_{0} b_{-1} \\
& -c a_{0} b_{-1}-c a_{-1} b_{0}, \\
R_{-2}= & \xi_{0} b_{-1}^{2}-c a_{-1} b_{-1}+\frac{1}{2} \lambda \varepsilon a_{-1}^{2}
\end{aligned}
$$

Solving this system of algebraic equations by using symbolic computation, we obtain the following results. 
Case 1. Consider

$$
\begin{gathered}
a_{1}=\frac{b_{1}\left(\varepsilon a_{-1}-4 \lambda \nu b_{-1}\right)}{\varepsilon b_{-1}}, \quad a_{0}=0, \quad a_{-1}=a_{-1}, \\
b_{1}=b_{1}, \quad b_{0}=0, \quad b_{-1}=b_{-1}, \\
\lambda=\lambda, \quad \varepsilon=\varepsilon, \quad \nu=\nu, \\
\xi_{0}=\frac{\lambda a_{-1}\left(\varepsilon a_{-1}-4 \lambda \nu b_{-1}\right)}{2 b_{-1}^{2}}, \quad c=\frac{\lambda\left(\varepsilon a_{-1}-2 \lambda \nu b_{-1}\right)}{b_{-1}},
\end{gathered}
$$

where $a_{-1}$ and $b_{-1}$ are free parameters which exist provided that $b_{-1} \neq 0$ and $\varepsilon a_{-1}-2 \lambda \nu b_{-1} \neq 0$. Substituting these results into (39), we obtain the following exact solution:

$$
\begin{gathered}
u(x, t) \\
=\frac{b_{1}\left(\varepsilon a_{-1}-4 \lambda \nu b_{-1}\right)}{\varepsilon b_{-1}} \exp \left(\lambda x-\frac{c t^{\alpha}}{\Gamma(1+\alpha)}\right) \\
+a_{-1} \exp \left(-\left(\lambda x-\frac{c t^{\alpha}}{\Gamma(1+\alpha)}\right)\right) \\
\quad \times\left(b_{1} \exp \left(\lambda x-\frac{c t^{\alpha}}{\Gamma(1+\alpha)}\right)\right. \\
\left.\quad+b_{-1} \exp \left(-\left(\lambda x-\frac{c t^{\alpha}}{\Gamma(1+\alpha)}\right)\right)\right)^{-1} .
\end{gathered}
$$

Case 2. Consider

$$
\begin{aligned}
& a_{1}=-\left(\varepsilon a_{-1}^{2} b_{0}^{2}-2 a_{-1} b_{0}^{2} \lambda \nu b_{-1}-2 \varepsilon a_{0} b_{0} b_{-1} a_{-1}\right. \\
&\left.+2 \lambda \nu a_{0} b_{0} b_{-1}^{2}+\varepsilon a_{0}^{2} b_{-1}^{2}\right) \\
& \times\left(\varepsilon a_{-1}-\lambda \nu b_{-1}\right) \\
& \times\left(4 b_{-1}^{2} \lambda^{2} \nu^{2}\right)^{-1}, \\
& b_{1}=-\varepsilon\left(\varepsilon a_{-1}^{2} b_{0}^{2}-2 a_{-1} b_{0}^{2} \lambda \nu b_{-1}-2 \varepsilon a_{0} b_{0} b_{-1} a_{-1}\right. \\
&\left.+2 \lambda \nu a_{0} b_{0} b_{-1}^{2}+\varepsilon a_{0}^{2} b_{-1}^{2}\right) \\
& \times\left(4 b_{-1}^{3} \lambda^{2} \nu^{2}\right)^{-1}, \\
& a_{0}= a_{0}, \quad a_{-1}=a_{-1}, \quad b_{0}=b_{0}, \\
& b_{-1}= b_{-1}, \quad \lambda=\lambda, \quad \varepsilon=\varepsilon, \quad \nu=\nu, \\
& \xi_{0}=\frac{\lambda a_{-1}\left(\varepsilon a_{-1}-2 \lambda \nu b_{-1}\right)}{2 b_{-1}^{2}}, \quad c=\frac{\lambda\left(\varepsilon a_{-1}-\lambda \nu b_{-1}\right)}{b_{-1}},
\end{aligned}
$$

where $a_{-1}, a_{0}, b_{-1}$, and $b_{0}$ are free parameters which exist provided that $b_{-1} \neq 0$ and $\varepsilon a_{-1}-2 \lambda \nu b_{-1} \neq 0$. Substituting these results into (39), we get the following exact solution:

$$
\begin{aligned}
& u(x, t) \\
& =-\left(\varepsilon a_{-1}^{2} b_{0}^{2}-2 a_{-1} b_{0}^{2} \lambda \nu b_{-1}-2 \varepsilon a_{0} b_{0} b_{-1} a_{-1}\right. \\
& \left.\quad+2 \lambda \nu a_{0} b_{0} b_{-1}^{2}+\varepsilon a_{0}^{2} b_{-1}^{2}\right)\left(\varepsilon a_{-1}-\lambda \nu b_{-1}\right)
\end{aligned}
$$

$$
\begin{aligned}
& \times\left(4 b_{-1}^{2} \lambda^{2} \nu^{2}\right)^{-1} \\
& \times \exp \left(\lambda x-\frac{c t^{\alpha}}{\Gamma(1+\alpha)}\right)+a_{0} \\
+ & a_{-1} \exp \left(-\left(\lambda x-\frac{c t^{\alpha}}{\Gamma(1+\alpha)}\right)\right) \\
\times & \left(-\varepsilon\left(\varepsilon a_{-1}^{2} b_{0}^{2}-2 a_{-1} b_{0}^{2} \lambda v b_{-1}-2 \varepsilon a_{0} b_{0} b_{-1} a_{-1}\right.\right. \\
& \left.+2 \lambda v a_{0} b_{0} b_{-1}^{2}+\varepsilon a_{0}^{2} b_{-1}^{2}\right) \\
& \times\left(4 b_{-1}^{3} \lambda^{2} \nu^{2}\right)^{-1} \\
& \times \exp \left(\lambda x-\frac{c t^{\alpha}}{\Gamma(1+\alpha)}\right)+b_{0} \\
& \left.+b_{-1} \exp \left(-\left(\lambda x-\frac{c t^{\alpha}}{\Gamma(1+\alpha)}\right)\right)\right)^{-1} .
\end{aligned}
$$

\section{Space-Time Fractional Cahn-Hilliard Equation}

We consider the space-time fractional Cahn-Hilliard equation [33]

$$
D_{t}^{\alpha} u-\gamma D_{x}^{\alpha} u-6 u\left(D_{x}^{\alpha} u\right)^{2}-\left(3 u^{2}-1\right) D_{x}^{2 \alpha} u+D_{x}^{4 \alpha} u=0,
$$

where $0<\alpha \leq 1$ and $u$ is the function of $(x, t)$. For the case corresponding to $\alpha=1$, this equation is related to a number of interesting physical phenomena like the spinodal decomposition, phase separation, and phase ordering dynamics. Moreover, it becomes important in material sciences [34]. Nevertheless we notice that this equation is very difficult to be solved and several articles investigated it [35].

Firstly, we consider the following transformations:

$$
u(x, t)=U(\xi), \quad \xi=\frac{x^{\alpha}}{\Gamma(1+\alpha)}-\frac{c t^{\alpha}}{\Gamma(1+\alpha)},
$$

where $c$ is a nonzero constant.

Substituting (47) into (46), we can know this equation reduced into an ODE:

$$
-c U^{\prime}-\gamma U^{\prime}-6 U\left(U^{\prime}\right)^{2}-3 U^{2} U^{\prime \prime}+U^{\prime \prime}+U^{\prime \prime \prime \prime}=0,
$$

where " $U$ " " $=d U / d \xi$.

Integrating (48) with respect to $\xi$ yields

$$
-c U-\gamma U-3 U^{2} U^{\prime}+U^{\prime}+U^{\prime \prime \prime}+\xi_{0}=0,
$$

where $\xi_{0}$ is a constant of integration.

Here take notice of nonlinear term in (49), and we can balance $U^{2} U^{\prime}$ and $U^{\prime \prime \prime}$ by the idea of the exp-function method 
[14] to determine the values of $p, q, c$, and $d$. By simple calculation, we have

$$
\begin{aligned}
U^{\prime \prime \prime} & =\frac{c_{1} \exp [(-7 p-c) \xi]+\cdots}{c_{2} \exp [-8 p \xi]+\cdots} \\
& =\frac{c_{1} \exp [-(7 p+c) \xi]+\cdots}{c_{2} \exp [-8 p \xi]+\cdots}, \\
U^{2} U^{\prime} & =\frac{c_{3} \exp [(-p-3 c) \xi]+\cdots}{c_{4} \exp [-4 p \xi]+\cdots} \\
& =\frac{c_{3} \exp [-(5 p+3 c) \xi]+\cdots}{c_{4} \exp [-8 p \xi]+\cdots},
\end{aligned}
$$

where $c_{i}$ are determined coefficients only for simplicity. Balancing the highest order of exp-function in (50), we have

$$
-(7 p+c)=-(5 c+3 p)
$$

which leads to the result that

$$
p=c
$$

Similarly, to determine the values of $d$ and $q$, we balance the linear term of lowest order in (49)

$$
\begin{aligned}
U^{\prime \prime \prime} & =\frac{\cdots+d_{1} \exp [(7 q+d) \xi]}{\cdots+d_{2} \exp [8 q \xi]}, \\
U^{2} U^{\prime} & =\frac{\cdots+d_{3} \exp [(2 d+6 q) \xi]}{\cdots+d_{4} \exp [8 q \xi]},
\end{aligned}
$$

where $d_{i}$ are determined coefficients only for simplicity. From (53), we obtain

$$
(6 q+2 d)=(d+7 q)
$$

and this gives

$$
q=d
$$

For simplicity, we set $p=c=1$ and $q=d=1$, so (11) reduces to

$$
u(\xi)=\frac{a_{1} \exp (\xi)+a_{0}+a_{-1} \exp (-\xi)}{b_{1} \exp (\xi)+b_{0}+b_{-1} \exp (-\xi)}
$$

Substituting (56) into (49) and by the help of symbolic computation, we obtain

$$
\begin{aligned}
& \frac{1}{A}\left[R_{4} \exp (4 \xi)+R_{3} \exp (3 \xi)+R_{2} \exp (2 \xi)+R_{1} \exp (\xi)\right. \\
& \quad+R_{0}+R_{-1} \exp (-\xi)+R_{-2} \exp (-2 \xi)+R_{-3} \exp (-3 \xi) \\
& \left.\quad+R_{-4} \exp (-4 \xi)\right]=0
\end{aligned}
$$

where

$$
\begin{aligned}
& A=\left(b_{-1} \exp (-\xi)+b_{0}+b_{1} \exp (\xi)\right)^{4}, \\
& R_{4}=\xi_{0} b_{1}^{4}-c a_{1} b_{1}^{3}-\gamma a_{1} b_{1}^{3}, \\
& R_{3}=-3 a_{1}^{3} b_{0}-2 a_{0} b_{1}^{3}-c a_{0} b_{1}^{3}-\gamma a_{0} b_{1}^{3}+2 a_{1} b_{1}^{2} b_{0} \\
& +3 a_{1}^{2} a_{0} b_{1}+4 \xi_{0} b_{1}^{3} b_{0}-3 \gamma a_{1} b_{1}^{2} b_{0}-3 c a_{1} b_{1}^{2} b_{0}, \\
& R_{2}=-10 a_{-1} b_{1}^{3}-6 a_{1}^{3} b_{-1}-c a_{-1} b_{1}^{3}-\gamma a_{-1} b_{1}^{3} \\
& +10 a_{1} b_{1}^{2} b_{-1}+6 a_{1}^{2} a_{-1} b_{1}-2 a_{1} b_{1} b_{0}^{2}+4 \xi_{0} b_{1}^{3} b_{-1} \\
& +2 a_{0} b_{1}^{2} b_{0}-6 a_{1}^{2} a_{0} b_{0}+6 a_{1} a_{0}^{2} b_{1}+6 \xi_{0} b_{1}^{2} b_{0}^{2} \\
& -3 c a_{1} b_{1} b_{0}^{2}-3 c a_{0} b_{1}^{2} b_{0}-3 \gamma a_{1} b_{1} b_{0}^{2}-3 \gamma a_{0} b_{1}^{2} b_{0} \\
& -3 c a_{1} b_{1}^{2} b_{-1}-3 \gamma a_{1} b_{1}^{2} b_{-1} \text {, } \\
& R_{1}=-3 c a_{0} b_{1} b_{0}^{2}-3 \gamma a_{0} b_{1} b_{0}^{2}-2 a_{0} b_{1} b_{0}^{2}-c a_{1} b_{0}^{3} \\
& -\gamma a_{1} b_{0}^{3}-3 a_{0}^{2} a_{1} b_{0}+4 \xi_{0} b_{1} b_{0}^{3}+2 a_{1} b_{0}^{3}+3 a_{0}^{3} b_{1} \\
& -6 c a_{1} b_{1} b_{0} b_{-1}-6 \gamma a_{1} b_{1} b_{0} b_{-1}+22 a_{0} b_{1}^{2} b_{-1} \\
& -10 a_{-1} b_{1}^{2} b_{0}-3 a_{1}^{2} a_{-1} b_{0}-15 a_{1}^{2} a_{0} b_{-1} \\
& -12 a_{1} b_{1} b_{0} b_{-1}-3 c a_{0} b_{1}^{2} b_{-1}-3 c a_{-1} b_{1}^{2} b_{0} \\
& -3 \gamma a_{0} b_{1}^{2} b_{-1}-3 \gamma a_{-1} b_{1}^{2} b_{0}+18 a_{1} b_{1} a_{0} a_{-1} \\
& +12 \xi_{0} b_{1}^{2} b_{0} b_{-1} \\
& R_{0}=-3 c a_{-1} b_{1} b_{0}^{2}-6 \gamma a_{0} b_{1} b_{0} b_{-1}+\xi_{0} b_{0}^{4}-c a_{0} b_{0}^{3} \\
& +28 a_{-1} b_{1}^{2} b_{-1}-28 a_{1} b_{-1}^{2} b_{1}+8 a_{1} b_{-1} b_{0}^{2} \\
& -6 c a_{0} b_{1} b_{0} b_{-1}-8 a_{-1} b_{1} b_{0}^{2} \\
& -3 c a_{1} b_{-1}^{2} b_{1}-3 c a_{1} b_{0}^{2} b_{-1}-3 c a_{-1} b_{1}^{2} b_{-1} \\
& -\gamma a_{0} b_{0}^{3}-3 \gamma a_{1} b_{-1}^{2} b_{1}-3 \gamma a_{1} b_{0}^{2} b_{-1}-3 \gamma a_{-1} b_{1}^{2} b_{-1} \\
& -3 \gamma a_{-1} b_{0}^{2} b_{1}+12 \xi_{0} b_{1} b_{0}^{2} b_{-1}-12 a_{1} a_{0}^{2} b_{-1} \\
& -12 a_{1}^{2} b_{-1} a_{-1}+12 a_{1} a_{-1}^{2} b_{1}+12 b_{1} a_{0}^{2} a_{-1} \\
& R_{-1}=-3 c a_{0} b_{-1} b_{0}^{2}-3 \gamma a_{0} b_{-1} b_{0}^{2}+2 a_{0} b_{-1} b_{0}^{2} \\
& -c a_{-1} b_{0}^{3}-\gamma a_{-1} b_{0}^{3}+3 a_{0}^{2} a_{-1} b_{0} \\
& +4 \xi_{0} b_{0}^{3} b_{-1}-2 a_{-1} b_{0}^{3}-3 a_{0}^{3} b_{-1}-6 c a_{-1} b_{1} b_{0} b_{-1} \\
& -6 \gamma a_{-1} b_{1} b_{0} b_{-1}+10 a_{1} b_{-1}^{2} b_{0}-22 a_{0} b_{-1}^{2} b_{1} \\
& +3 a_{1} a_{-1}^{2} b_{0}+15 a_{0} a_{-1}^{2} b_{1}-12 a_{-1} b_{1} b_{0} b_{-1} \\
& -3 c a_{1} b_{0} b_{-1}^{2}-3 c a_{0} b_{1} b_{-1}^{2}-3 \gamma a_{1} b_{-1}^{2} b_{0} \\
& -3 \gamma a_{0} b_{1} b_{-1}^{2}-18 a_{1} a_{-1} a_{0} b_{-1}+12 \xi_{0} b_{-1}^{2} b_{0} b_{1} \text {, }
\end{aligned}
$$




$$
\begin{aligned}
R_{-2}= & 10 a_{1} b_{-1}^{3}+6 a_{-1}^{3} b_{1}-c a_{1} b_{-1}^{3}-\gamma a_{1} b_{-1}^{3} \\
& -10 a_{-1} b_{1} b_{-1}^{2}-6 a_{1} a_{-1}^{2} b_{-1}+4 \xi_{0} b_{-1}^{3} b_{1}-2 a_{0} b_{0} b_{-1}^{2} \\
& +2 a_{-1} b_{0}^{2} b_{-1}+6 a_{0} a_{-1}^{2} b_{0}-6 a_{-1} a_{0}^{2} b_{-1}+6 \xi_{0} b_{-1}^{2} b_{0}^{2} \\
& -3 c a_{0} b_{0} b_{-1}^{2}-3 c a_{-1} b_{-1} b_{0}^{2}-3 \gamma a_{0} b_{0} b_{-1}^{2} \\
& -3 \gamma a_{-1} b_{0}^{2} b_{-1}-3 c a_{-1} b_{-1}^{2} b_{1}-3 \gamma a_{-1} b_{-1}^{2} b_{1}, \\
R_{-3}= & 2 a_{0} b_{-1}^{3}+3 a_{-1}^{3} b_{0}-c a_{0} b_{-1}^{3}-\gamma a_{0} b_{-1}^{3}-2 a_{-1} b_{0} b_{-1}^{2} \\
& -3 a_{-1}^{2} a_{0} b_{-1}+4 \xi_{0} b_{0} b_{-1}^{3}-3 c a_{-1} b_{0} b_{-1}^{2} \\
& -3 \gamma a_{-1} b_{0} b_{-1}^{2}, \\
R_{-4}= & \xi_{0} b_{-1}^{4}-c a_{-1} b_{-1}^{3}-\gamma a_{-1} b_{-1}^{3} .
\end{aligned}
$$

Solving this system of algebraic equations by using symbolic computation, we obtain the following results:

$$
\begin{aligned}
a_{1}=\frac{a_{0}^{2} \sqrt{6}}{8 b_{-1}}, & a_{0}=a_{0}, \quad a_{-1}=b_{-1} \sqrt{\frac{2}{3}}, \\
b_{1}=\frac{3 a_{0}^{2}}{8 b_{-1}}, & b_{0}=-a_{0} \sqrt{6}, \quad b_{-1}=b_{-1}, \\
c=c, & \gamma=-c, \quad \xi_{0}=0,
\end{aligned}
$$

where $a_{0}$ and $b_{-1} \neq 0$ are free parameters.

From (59), substituting these results into (56), we obtain the following exact solution:

$$
\begin{aligned}
& u(x, t) \\
& =\frac{a_{0}^{2} \sqrt{6}}{8 b_{-1}} \exp \left(\frac{x^{\alpha}}{\Gamma(1+\alpha)}-\frac{c t^{\alpha}}{\Gamma(1+\alpha)}\right)+a_{0} \\
& +b_{-1} \sqrt{\frac{2}{3}} \exp \left(-\left(\frac{x^{\alpha}}{\Gamma(1+\alpha)}-\frac{c t^{\alpha}}{\Gamma(1+\alpha)}\right)\right) \\
& \quad \times\left(\frac{3 a_{0}^{2}}{8 b_{-1}} \exp \left(\frac{x^{\alpha}}{\Gamma(1+\alpha)}-\frac{c t^{\alpha}}{\Gamma(1+\alpha)}\right)-a_{0} \sqrt{6}\right. \\
& \left.\quad+b_{-1} \exp \left(-\left(\frac{x^{\alpha}}{\Gamma(1+\alpha)}-\frac{c t^{\alpha}}{\Gamma(1+\alpha)}\right)\right)\right)^{-1} .
\end{aligned}
$$

\section{Conclusion}

In this paper, we have successfully developed fractional complex transform with the help of exp-function method to obtain exact solution of some fractional differential equations. The fractional complex transform and exp-function methods are extremely simple but effective and powerful for solving fractional differential equations. These methods are accessible to solve other similar nonlinear equations in fractional calculus. To our knowledge, these new solutions have not been reported in former literature; they may be of significant importance for the explanation of some special physical phenomena.

\section{Conflict of Interests}

The authors declare that there is no conflict of interests in this paper.

\section{References}

[1] K. B. Oldham and J. Spanier, The Fractional Calculus, Akademic Press, New York, NY, USA, 1974.

[2] K. S. Miller and B. Ross, An Introduction to the Fractional Calculus and Fractional Differential Equations, A Wiley-Interscience Publication, John Wiley \& Sons, New York, NY, USA, 1993.

[3] I. Podlubny, Fractional differential equations, vol. 198 of Mathematics in Science and Engineering, Academic Press, San Diego, CA, USA, 1999.

[4] A. A. Kilbas, H. M. Srivastava, and J. J. Trujillo, Theory and Applications of Fractional Differential Equations, Elsevier, Amsterdam, The Netherlands, 2006.

[5] S. Zhang, Q.-A. Zong, D. Liu, and Q. Gao, "A generalized expfunction method for fractional riccati differential equations," Communications in Fractional Calculus, vol. 1, no. 1, pp. 48-51, 2010.

[6] S. Zhang and H.-Q. Zhang, "Fractional sub-equation method and its applications to nonlinear fractional PDEs," Physics Letters A, vol. 375, no. 7, pp. 1069-1073, 2011.

[7] B. Tang, Y. He, L. Wei, and X. Zhang, "A generalized fractional sub-equation method for fractional differential equations with variable coefficients," Physics Letters A, vol. 376, no. 38-39, pp. 2588-2590, 2012.

[8] S. Guo, L. Mei, Y. Li, and Y. Sun, "The improved fractional sub-equation method and its applications to the space-time fractional differential equations in fluid mechanics," Physics Letters A, vol. 376, no. 4, pp. 407-411, 2012.

[9] B. Zheng, " $\left(G^{\prime} / G\right)$-expansion method for solving fractional partial differential equations in the theory of mathematical physics," Communications in Theoretical Physics, vol. 58, no. 5, pp. 623-630, 2012.

[10] K. A. Gepreel and S. Omran, "Exact solutions for nonlinear partial fractional differential equations," Chinese Physics B, vol. 21, no. 11, Article ID 110204, 2012.

[11] B. Lu, "The first integral method for some time fractional differential equations," Journal of Mathematical Analysis and Applications, vol. 395, no. 2, pp. 684-693, 2012.

[12] J.-H. He, S. K. Elagan, and Z. B. Li, "Geometrical explanation of the fractional complex transform and derivative chain rule for fractional calculus," Physics Letters A, vol. 376, no. 4, pp. 257259, 2012.

[13] R. W. Ibrahim, "Fractional complex transforms for fractional differential equations," Advances in Difference Equations, vol. 2012, article 192, 2012

[14] J.-H. He and X.-H. Wu, "Exp-function method for nonlinear wave equations," Chaos, Solitons \& Fractals, vol. 30, no. 3, pp. 700-708, 2006.

[15] S. Zhang, "Application of Exp-function method to highdimensional nonlinear evolution equation," Chaos, Solitons and Fractals, vol. 38, no. 1, pp. 270-276, 2008.

[16] A. Bekir and A. C. Cevikel, "New solitons and periodic solutions for nonlinear physical models in mathematical physics," Nonlinear Analysis. Real World Applications, vol. 11, no. 4, pp. 3275-3285, 2010. 
[17] S. A. El-Wakil, M. A. Madkour, and M. A. Abdou, "Application of Exp-function method for nonlinear evolution equations with variable coefficients," Physics Letters A, vol. 369, no. 1-2, pp. 6269, 2007.

[18] S. D. Zhu, "Exp-function method for the Hybrid-Lattice system," International Journal of Nonlinear Sciences and Numerical Simulation, vol. 8, no. 3, pp. 461-464, 2007.

[19] A. Bekir, "Application of the exp-function method for nonlinear differential-difference equations," Applied Mathematics and Computation, vol. 215, no. 11, pp. 4049-4053, 2010.

[20] C. Q. Dai and J. L. Chen, "New analytic solutions of stochastic coupled KdV equations," Chaos, Solitons \& Fractals, vol. 42, no. 4, pp. 2200-2207, 2009.

[21] M. Caputo, "Linear models of dissipation whose Q is almost frequency independent II," Geophysical Journal International, vol. 13, no. 5, pp. 529-539, 1967.

[22] S. G. Samko, A. A. Kilbas, and O. I. Marichev, Fractional integrals and derivatives, Gordon and Breach Science Publishers, Switzerland, 1993.

[23] G. Jumarie, "Modified Riemann-Liouville derivative and fractional Taylor series of nondifferentiable functions further results," Computers \& Mathematics with Applications, vol. 51, no. 9-10, pp. 1367-1376, 2006.

[24] G. Jumarie, "Table of some basic fractional calculus formulae derived from a modified Riemann-Liouville derivative for nondifferentiable functions," Applied Mathematics Letters, vol. 22, no. 3, pp. 378-385, 2009.

[25] Z.-B. Li and J.-H. He, "Fractional complex transform for fractional differential equations," Mathematical \& Computational Applications, vol. 15, no. 5, pp. 970-973, 2010.

[26] Z.-B. Li and J.-H. He, "Application of the fractional complex transform to fractional differential equations," Nonlinear Science Letters A, vol. 2, pp. 121-126, 2011.

[27] J.-H. He and M. A. Abdou, "New periodic solutions for nonlinear evolution equations using exp-function method," Chaos, Solitons \& Fractals, vol. 34, no. 5, pp. 1421-1429, 2007.

[28] A. Ebaid, "Exact solitary wave solutions for some nonlinear evolution equations via exp-function method," Physics Letters A, vol. 365, no. 3, pp. 213-219, 2007.

[29] A. Bekir, "The exp-function method for Ostrovsky equation," International Journal of Nonlinear Sciences and Numerical Simulation, vol. 10, no. 6, pp. 735-739, 2009.

[30] A. M. A. El-Sayed, S. Z. Rida, and A. A. M. Arafa, "Exact solutions of fractional-order biological population model," Communications in Theoretical Physics, vol. 52, no. 6, pp. 992996, 2009.

[31] B. Lu, "Bäcklund transformation of fractional Riccati equation and its applications to nonlinear fractional partial differential equations," Physics Letters A, vol. 376, no. 28-29, pp. 2045-2048, 2012.

[32] M. Inc, "The approximate and exact solutions of the spaceand time-fractional Burgers equations with initial conditions by variational iteration method," Journal of Mathematical Analysis and Applications, vol. 345, no. 1, pp. 476-484, 2008.

[33] H. Jafari, H. Tajadodi, N. Kadkhoda, and D. Baleanu, "Fractional subequation method for Cahn-Hilliard and KleinGordon equations," Abstract and Applied Analysis, vol. 2013, Article ID 587179, 5 pages, 2013.

[34] S. M. Choo, S. K. Chung, and Y. J. Lee, "A conservative difference scheme for the viscous Cahn-Hilliard equation with a nonconstant gradient energy coefficient," Applied Numerical Mathematics, vol. 51, no. 2-3, pp. 207-219, 2004.
[35] J. Kim, "A numerical method for the Cahn-Hilliard equation with a variable mobility," Communications in Nonlinear Science and Numerical Simulation, vol. 12, no. 8, pp. 1560-1571, 2007. 


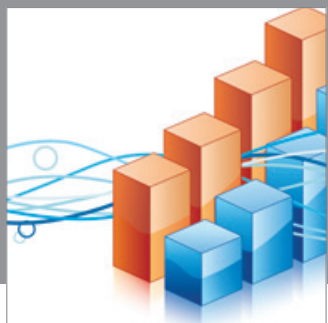

Advances in

Operations Research

mansans

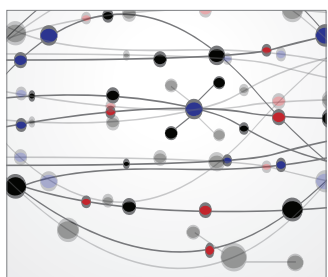

The Scientific World Journal
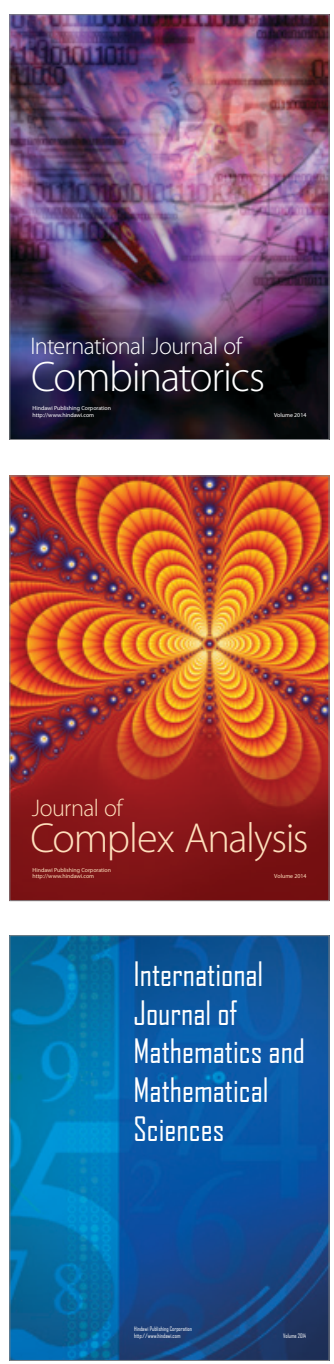
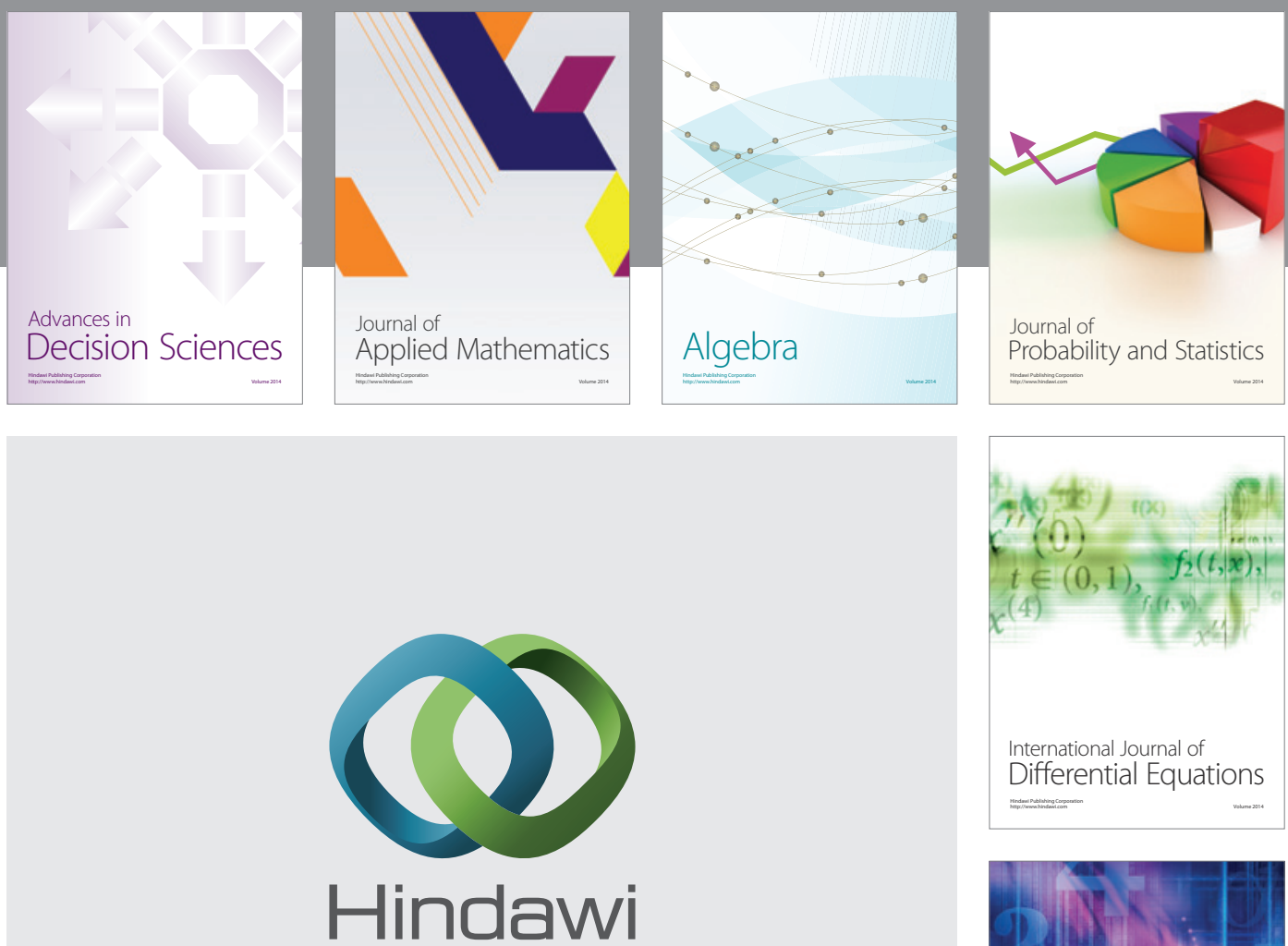

Submit your manuscripts at http://www.hindawi.com
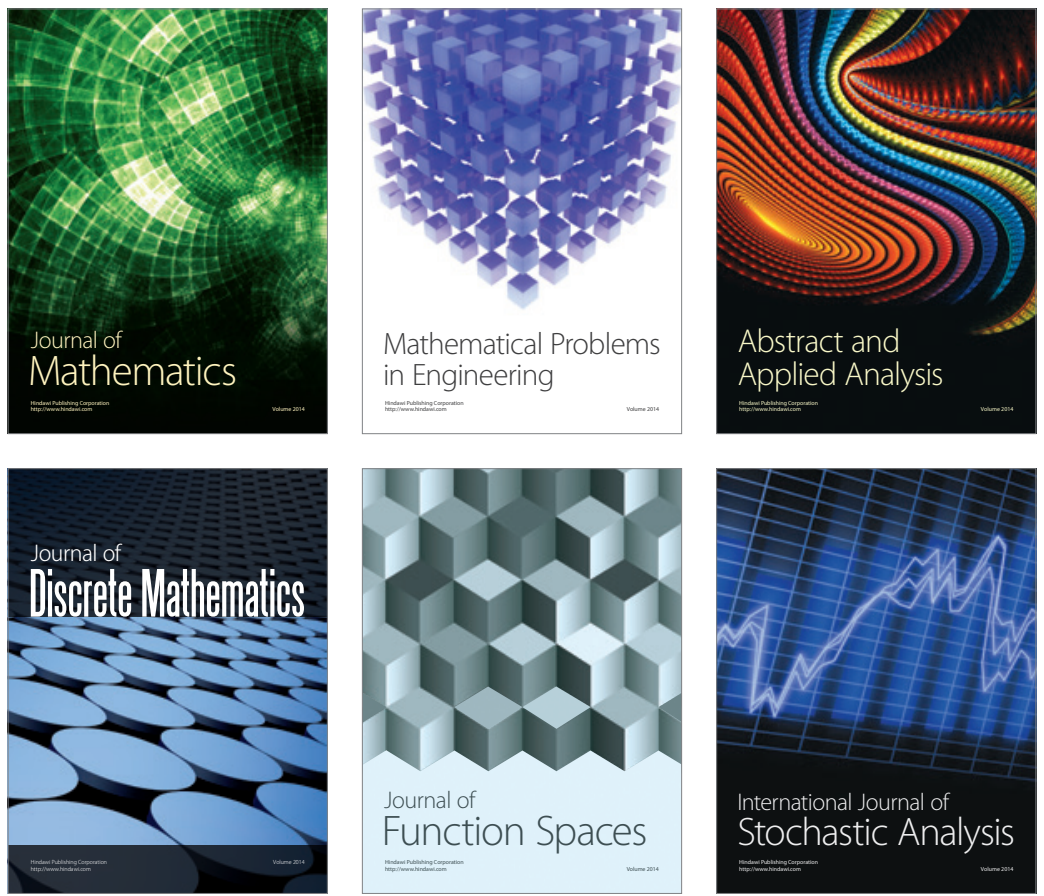

Journal of

Function Spaces

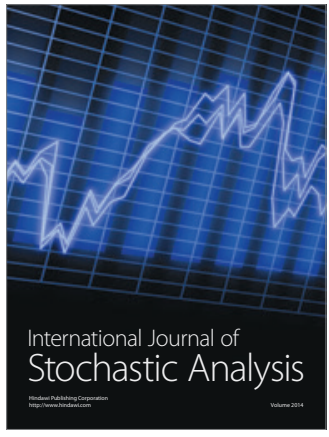

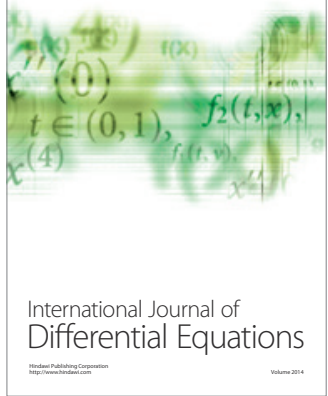
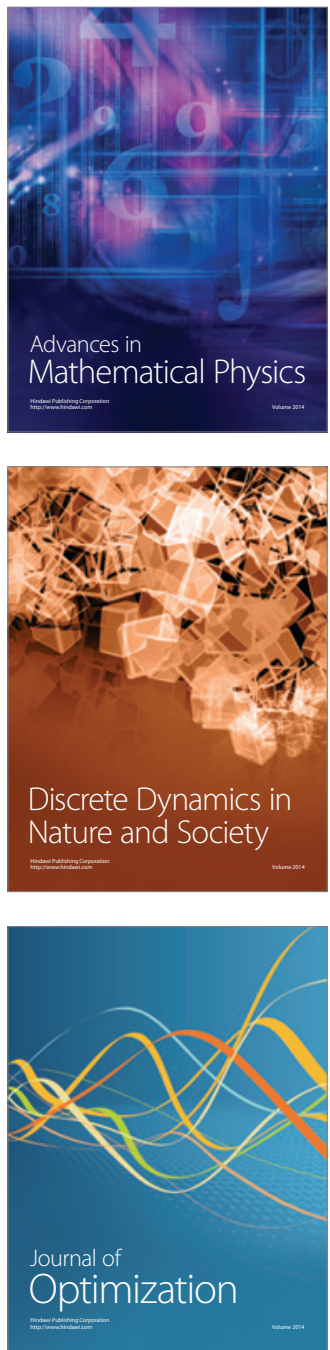